\title{
Computer vision-based image analysis for the estimation of acrylamide concentrations of potato chips and french fries
}

\author{
V. Gökmen ${ }^{\mathrm{a}, *}$, H.Z. Şenyuva ${ }^{\mathrm{b}}$, B. Dülek ${ }^{\mathrm{c}}$, A.E. Çetin ${ }^{\mathrm{c}}$ \\ ${ }^{a}$ Department of Food Engineering, Hacettepe University, 06532 Beytepe, Ankara, Turkey \\ b Ankara Test and Analysis Laboratory, Scientific and Technical Research Council of Turkey, Ankara 06330, Turkey \\ ${ }^{\mathrm{c}}$ Department of Electrical and Electronics Engineering, Bilkent University, 06533 Bilkent, Ankara, Turkey
}

Received 13 June 2005; received in revised form 7 February 2006; accepted 7 February 2006

\begin{abstract}
In this study, digital colour images of fried potato chips and french fries were analyzed to estimate acrylamide levels based on the correlation with analyses using liquid chromatography-mass spectrometry. In fried potato images, bright yellow (Region 1), yellowish brown (Region 2) and darker brown (Region 3) regions were clearly visible, having different kinds of image pixels with characteristic mean values of red, green and blue components. Pixels of the fried potato image were classified into three sets (Set 1, Set 2 and Set 3 ) by means of semi-automatic and automatic segmentation. There was a strong correlation between acrylamide concentration and NA2 value, which is defined as the number of pixels in Set 2 divided by the total number of pixels of the entire fried potato image. To verify the applicability of this approach, a linear regression equation was used to estimate the acrylamide concentrations of a number of commercial potato chips and home-made french fries. Mean differences between the measured and predicted acrylamide concentrations were found to be $+4 \pm 14 \%$ and $-14 \pm 24 \%$ for commercial potato chips and home-made french fries, respectively.
\end{abstract}

(c) 2006 Elsevier Ltd. All rights reserved.

Keywords: Acrylamide; Potato chips; French fries; Surface colour; Computer vision-based image analysis

\section{Introduction}

Acrylamide formation was found to occur during the browning process, by Maillard reaction, of reducing sugars with asparagine at temperatures above $120^{\circ} \mathrm{C}$ (Friedman, 2003; Mottram, Wedzicha, \& Dodson, 2002; Stadler et al., 2002; Yaylayan, Wnorowski, \& Locas, 2003). Coloured products are also formed in foods during heating as a result of the Maillard reaction and melanoidins are known to be the main end-products of the reaction (Márquez \& Aňón, 1986; Pedrechi, Moyano, Kaack, \& Granby, 2005; Şenyuva \& Gökmen, 2005). These brown polymers have significant effects on the quality of food, since colour is an important food attribute and a key factor in consumer acceptance. The mechanism of formation of brown colour

\footnotetext{
* Corresponding author. Fax: +90 3122992123.

E-mail address: vgokmen@hacettepe.edu.tr (V. Gökmen).
}

is not fully understood and the structure of melanoidins is largely unknown (Martins \& van Boekel, 2003).

Since colour can easily be measured, it may be used as an indicator of other Maillard reaction products, such as acrylamide. Colour of foods is usually measured in by the $L^{*} a^{*} b^{*}$ system, which is an international standard for colour measurements, adopted by the Commission Internationale d'Eclairage (CIE) in 1976. $L^{*}$ is the luminance or lightness component (black to white), and parameters $a^{*}$ (from green to red) and $b^{*}$ (from blue to yellow) are the two chromatic components (Papadakis, Abdul-Malek, Kamdem, \& Yam, 2000). Amrein, Schönbächler, Escher, and Amado (2004) reported a significant correlation between the $L^{*}$ values and the acrylamide content during baking at $180^{\circ} \mathrm{C}$. Surdyk, Rosén, Andersson, and Åman (2004) also reported a highly significant correlation between colour and acrylamide content in bread crust during baking. Pedrechi et al. (2005) reported that $L^{*}$ and $b^{*}$ 
values did not show as extensive changes as those shown by $a^{*}$ during frying of potato chips. They found a linear correlation between the acrylamide concentration and the colour of potato chips, represented by the redness component $a^{*}$ at temperatures of 120,150 and $180^{\circ} \mathrm{C}$ for up to $5 \mathrm{~min}$ of frying. Şenyuva and Gökmen (2005) reported that the changes in acrylamide levels and CIE $a^{*}$ followed almost the same trend during heating of coffee at 150, 200 and $225^{\circ} \mathrm{C}$.

Although the previous findings profoundly suggest that surface colour may be correlated with acrylamide concentration in thermally processed foods, the measurement of surface image and its colour properties need to be investigated in more detail to establish a useful correlation. This paper describes a computer vision-based image analysis for the estimation of acrylamide levels of fried potatoes.

\section{Materials and methods}

\subsection{Chemicals and consumables}

Acrylamide (99+\%) and ${ }^{13} \mathrm{C}_{3}$-labelled acrylamide (99\% isotopic purity) were obtained from Sigma (Diesenhofen, Germany) and Cambridge Isotope Laboratories (Andover, MA, USA), respectively. Methanol, potassium hexacyanoferrate, zinc sulfate, formic acid $(98 \%)$ and acetic acid (glacial) were of analytical grade and obtained from Merck (Darmstadt, Germany). Ultra pure water was used throughout the experiments (MilliQ system, Millipore, Bedford, MA, USA). Oasis HLB (1 ml, $30 \mathrm{mg})$ SPE cartridges were supplied by Waters (Milford, MA, USA). Glass vials with septum screw caps were supplied by Agilent Technologies (Wilmington, DE, USA). The analytical column (Inertsil ODS-3, $250 \times 4.6 \mathrm{~mm}, 5 \mu \mathrm{m}$ ) was supplied by HiChrom (Berkshire, England).

Stock solutions of acrylamide $(1 \mathrm{mg} / \mathrm{ml})$ and ${ }^{13} \mathrm{C}_{3^{-}}$labelled acrylamide $(0.1 \mathrm{mg} / \mathrm{ml})$ were prepared by dissolving in distilled water. Working standards were prepared by diluting the stock solution of acrylamide to concentrations of $0.1,0.2,0.3,0.5,1.0$ and $2.0 \mu \mathrm{g} / \mathrm{ml}$ with $0.01 \mathrm{mM}$ acetic acid. Stock solutions and working standards were kept at $4{ }^{\circ} \mathrm{C}$ for one month. Carrez I solution was prepared by dissolving $15 \mathrm{~g}$ of potassium hexacyanoferrate in $100 \mathrm{ml}$ of water, and Carrez II solution by dissolving $30 \mathrm{~g}$ of zinc sulfate in $100 \mathrm{ml}$ of water.

\subsection{Preparation of fried potatoes}

Ten types of potato tubers, obtained from local markets, were used to prepare potato chips or french fries. Potatoes were washed and peeled before cutting. One type of potato tuber was used to prepare potato chips $(2 \mathrm{~mm})$, using a slicer. Frying was performed in an oil bath set at $170^{\circ} \mathrm{C}$ with sampling at 1, 3, 5, 8, 10, 15, 30, 45 and $60 \mathrm{~min}$ for potato chips. Formations of acrylamide and colour were monitored in potato chips in a time-dependent manner. The data were used to build correlations between acrylamide levels and surface image properties for fried potatoes. In order to test the applicability of the computer vision-based approach, french fries made of 10 types of tubers were analyzed. Potatoes cut into strips $(8.5 \times 8.5 \times 70.0 \mathrm{~mm})$ were fried at $170{ }^{\circ} \mathrm{C}$ for $10 \mathrm{~min}$ in an oil bath.

\subsection{Measurement of acrylamide}

\subsubsection{Sample preparation}

A sample preparation procedure previously, described by us elsewhere was used (Gökmen, Şenyuva, Acar, \& Sarıoğlu, 2005). Finely ground potato chips or french fries were weighed $(1 \mathrm{~g})$ into a $10 \mathrm{ml}$ glass centrifuge tube with cap. The sample was suspended in $5 \mathrm{ml}$ of methanol and extracted for $2 \mathrm{~min}$ in a vortex mixer. The suspension was centrifuged at $5000 \mathrm{rpm}$ for $10 \mathrm{~min}$. The clear supernatant was transferred into a centrifuge tube and treated with Carrez I and II solutions ( $25 \mu \mathrm{l}$ each) to precipitate the coextractives. Following centrifugation at $5000 \mathrm{rpm}$ for $5 \mathrm{~min}, 1.0 \mathrm{ml}$ of clear supernatant ( $0.2 \mathrm{~g}$ sample) was quantitatively transferred into a conical bottom glass test tube placed in a water bath at $40{ }^{\circ} \mathrm{C}$ and evaporated to dryness under nitrogen at $3 \mathrm{psig}$. The remaining residue was immediately redissolved in $1 \mathrm{ml}$ of water by mixing in a vortex mixer for $1 \mathrm{~min}$. For the SPE cleanup, an Oasis HLB cartridge was preconditioned, consequently, with $1 \mathrm{ml}$ of methanol and $1 \mathrm{ml}$ of water at a rate of two drops per second, using a syringe. Then, $1 \mathrm{ml}$ of the extract was passed through the cartridge at a rate of one drop per second using a syringe. The first 10 drops of the effluent were discarded to prevent any dilution of sample by replacing water hold in the sorbent void fraction with the sample effluent. The subsequent drops were collected and filtered through a $0.45 \mu \mathrm{m}$ syringe filter and $20 \mu \mathrm{l}$ of the final test solution were injected onto the LC column for quantitation by LC-MS.

\subsection{2. $L C-M S$ analysis}

LC-MS analyses were performed by an Agilent 1100 HPLC system, consisting of a binary pump, an autosampler and a temperature-controlled column oven, coupled to an Agilent $1100 \mathrm{MS}$ detector equipped with atmospheric pressure chemical ionization interface. The analytical separation was performed on a Inertsil ODS-3 column $(250 \times 4.6 \mathrm{~mm}, 5 \mu \mathrm{m})$, using an isocratic mixture of $0.01 \mathrm{mM}$ acetic acid in $0.2 \%$ aqueous solution of formic acid at a flow rate of $0.6 \mathrm{ml} / \mathrm{min}$ at $25^{\circ} \mathrm{C}$. The LC eluent was directed to the MS system after a delay time of $6.5 \mathrm{~min}$, using MSD software. Data acquisition was performed in selected ion monitoring mode, using the interface parameters: drying gas $\left(\mathrm{N}_{2}, 100 \mathrm{psig}\right)$ flow of $4 \mathrm{l} / \mathrm{min}$, nebulizer pressure of $60 \mathrm{psig}$, drying gas temperature of $325^{\circ} \mathrm{C}$, vaporizer temperature of $425^{\circ} \mathrm{C}$, capillary voltage of $4 \mathrm{kV}$, corona current of $4 \mu \mathrm{A}$, fragmentor voltage of $55 \mathrm{eV}$. Ions monitored were $\mathrm{m} / \mathrm{z} 72$ and 55 for acrylamide and $m / z 75$ and 58 for ${ }^{13} \mathrm{C}_{3}$-labelled acrylamide for the quantification of acrylamide in the samples. 


\subsection{Measurement of CIE colour parameters}

Colour measurements $\left(L^{*} a^{*} b^{*}\right.$ parameters) were performed using a Minolta CM-3600d model spectrophotometer. Potato chips and french fries were aligned to sample measurement hole, manually, to measure the reflectance from both front and rear sides.

\section{Results and discussion}

\subsection{Formation of colour and acrylamide in potato chips during frying}

In potato chips, measured acrylamide concentration increased rapidly at the onset of frying, reaching an apparent maximum concentration of $5482 \mathrm{ng} / \mathrm{g}$ at $8 \mathrm{~min}$. Then the acrylamide concentration in potato chips tended to decrease exponentially. These results suggest that acrylamide forms as an intermediate product during Maillard reaction and its concentration begins to decrease as the rate of degradation exceeds the rate of formation during heating. As illustrated in Fig. 1, change of colour in potato chips is a dynamic process in which certain colour transitions occur as the frying proceeds. The initial pale soft yellow colour of potato first turned to bright yellow, then to brownish yellow during $8-10 \mathrm{~min}$ of frying at $170{ }^{\circ} \mathrm{C}$. After $10 \mathrm{~min}$, browning in the surface became clearer, reaching a darkbrown at the end of frying for $60 \mathrm{~min}$. CIE colour space parameters $\left(L^{*} a^{*} b^{*}\right)$ were determined to describe the changes occurring in the colour of potato chips during frying. CIE $L^{*}$ and $b^{*}$ values decreased exponentially during frying at $170{ }^{\circ} \mathrm{C}$ while CIE $a^{*}$ value increased rapidly during the first 5-8 min of frying reaching a plateau at ca. $15 \mathrm{~min}$ of frying. It tended to decrease as the frying proceeded.

As seen in Fig. 1, the change of redness parameter $a^{*}$ during frying was somewhat similar to that of acrylamide concentration in potato chips. However, these two variables could not be linearly correlated with each other. Here, acrylamide concentrations were translated to a dimension-
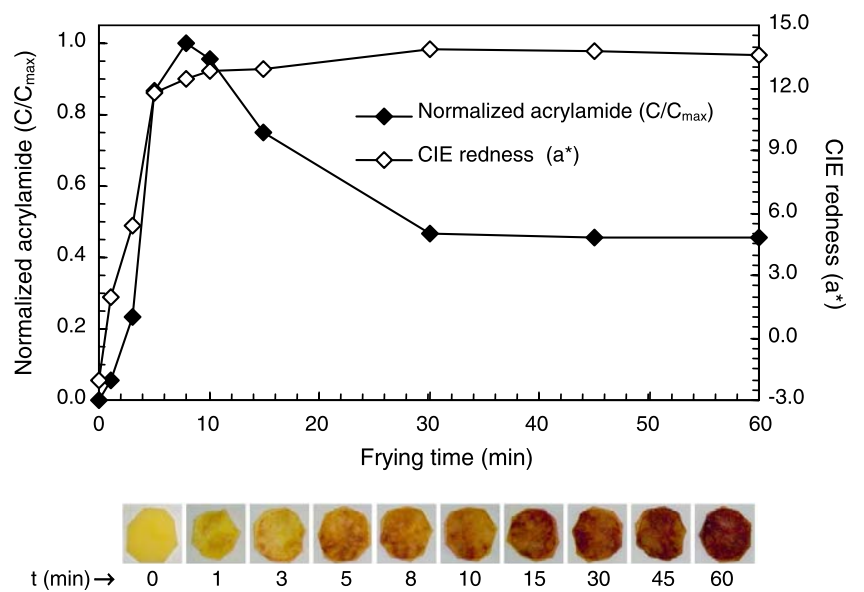

Fig. 1. Change of acrylamide concentration and CIE redness parameter $\mathrm{a}^{*}$ in potato chips during frying at $170^{\circ} \mathrm{C}$. less form by dividing each concentration by the apparent maximum concentration of acrylamide $(5482 \mathrm{ng} / \mathrm{g})$ measured in potato chips during the frying process.

Pedrechi et al. (2005) reported that CIE $L^{*}$ and $b^{*}$ parameters did not show as extensive changes as those shown by the CIE $a^{*}$ parameter during frying of potato chips. A linear correlation was found between the acrylamide concentration and the colour of potato chips, represented by the redness component $a^{*}$ at temperatures of 120,150 and $180{ }^{\circ} \mathrm{C}$ for up to $5 \mathrm{~min}$ of frying. However, the effect of prolonged frying on acrylamide concentration and colour was not mentioned by these researchers. Taubert, Harlfinger, Henkes, Berkels, and Schömig (2004) investigated the relation between the level of surface browning and acrylamide concentration of french fries by linear regression. They reported that there could be a close correlation for smallsurface material being fried. A somewhat less close correlation was observed for intermediate-surface material, while no correlation was observed for large-surface material.

Since the acrylamide concentrations were lower in darker potato chips, CIE $L^{*} a^{*} b^{*}$ parameters used to measure nonhomogeneous surface colour may not be a reliable predictor of acrylamide concentration in potato chips. Image analysis, instead of colour analysis, may provide a solution to estimate the acrylamide level in a given potato chip image.

\subsection{Computer vision-based analysis of fried potato images}

It is experimentally observed that a machine vision based system can be designed to remove fried potatoes having high acrylamide levels from a packaging line. It may not be possible to define a specific range of colours for acrylamide level estimation in the CIE $L^{*} a^{*} b^{*}$ colour space. However, after the frying process, three different kinds of image pixels appear in a typical potato image, as can be observed in Fig. 2a. Digital image pixel values of a potato chip can be used to estimate the acrylamide levels in a fried potato chip. In this section, a relationship between the acrylamide concentration in a fried potato chip and its image is established.

A typical image captured by a digital camera consists of an array of vectors called pixels. Each pixel has red, green and blue colour values:

$$
x[n, m]=\left[\begin{array}{l}
x_{\mathrm{r}}(n, m) \\
x_{\mathrm{g}}(n, m) \\
x_{\mathrm{b}}(n, m)
\end{array}\right]
$$

where $x_{\mathrm{r}}(n, m), x_{\mathrm{g}}(n, m)$ and $x_{\mathrm{b}}(n, m)$ are values of the red, green and blue components of the $(m, n)$ th pixel, respectively. In digital images, $x_{\mathrm{r}}, x_{\mathrm{g}}$ and $x_{\mathrm{b}}$ colour components are represented in 8 bits, i.e., they are allowed to take integer values between 0 and $255\left(=2^{8}-1\right)$ (Gonzales \& Woods, 2002). Digital and analogue cameras have built-in whitebalancing systems modifying actual colour values, therefore, pixel values in an image, captured by a camera of a machine vision system or a consumer camera, may not correspond to true colours of imaged objects. In addition, 


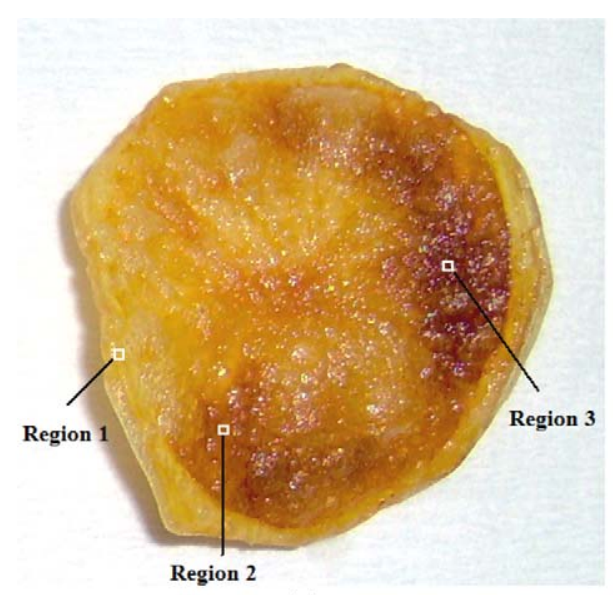

(a)

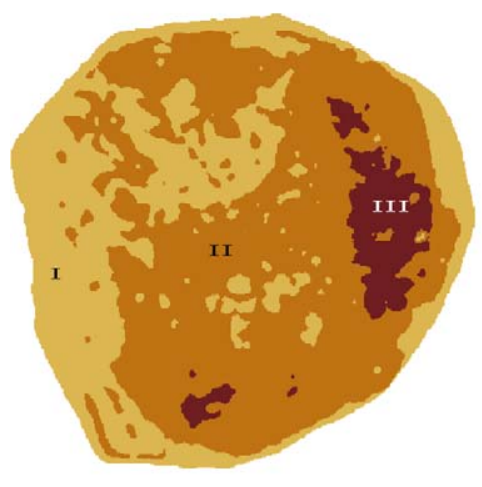

(b)

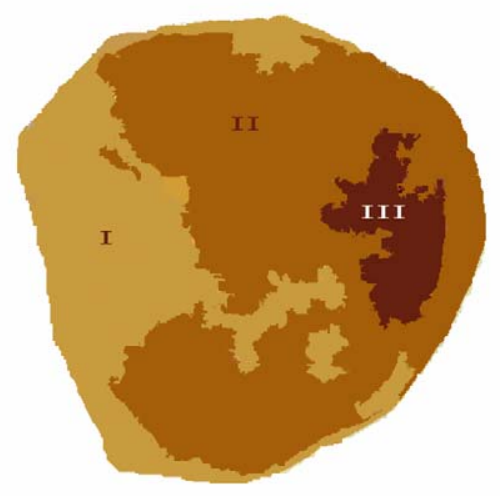

(c)

Fig. 2. (a) Original fried potato chip image with cropped windows shown; (b) result of semi-automatic segmentation algorithm; and (c) result of automatic mean shift segmentation algorithm.

CCD or CMOS imaging sensors of some cameras may not be calibrated during production. As mentioned above, even though it may not be possible to define a specific range of colours for acrylamide estimation in the CIE $L^{*} a^{*} b^{*}$ colour space, one can clearly visualize three different colours (or equivalently three different kinds of pixels) in a fried potato chip image as shown in Fig. 2a.

Three small rectangular windows were manually marked in Fig. 2a. Pixel values corresponding to small rectangular windows are given in Table 1. It was experimentally observed that Region 2 had a high probability of containing the highest level of acrylamide. These windows were cropped from the image, as shown in Fig. 2a, using the "imcrop" command of MATLAB.

Image pixel values corresponding to Region 1 have a mean value of [198.43 159.5365 .12$]$, i.e., the mean value of the red, green and blue components are 198.43, 159.53 , and 65.12 , respectively. Average image pixel values corresponding to Region 2 and Region 3 are [189.61 114.6 1.4] and [103.56 41.75 26.18], respectively. Pixels of the fried potato image shown in Fig. 2a can be classified into three sets, based on their Euclidian distances to the above three representative mean values. Members of Set 2 in Fig. $2 \mathrm{~b}$ are those pixels having the property:

$$
\begin{aligned}
& \left\{\left(x_{\mathrm{r}}(n, m)-189.61\right)^{2}+\left(x_{\mathrm{g}}(n, m)-114.6\right)^{2}+\left(x_{\mathrm{b}}(n, m)-1.4\right)^{2}\right\} \\
& \leqslant\left\{\left(x_{\mathrm{r}}(n, m)-198.43\right)^{2}+\left(x_{\mathrm{g}}(n, m)-159.53\right)^{2}\right. \\
& \left.\quad+\left(x_{\mathrm{b}}(n, m)-65.12\right)^{2}\right\}
\end{aligned}
$$

and

$$
\begin{aligned}
& \left\{\left(x_{\mathrm{r}}(n, m)-189.61\right)^{2}+\left(x_{\mathrm{g}}(n, m)-114.6\right)^{2}+\left(x_{\mathrm{b}}(n, m)-1.4\right)^{2}\right\} \\
& \leqslant\left\{\left(x_{\mathrm{r}}(n, m)-103.56\right)^{2}+\left(x_{\mathrm{g}}(n, m)-41.75\right)^{2}\right. \\
& \left.\quad+\left(x_{\mathrm{b}}(n, m)-26.18\right)^{2}\right\}
\end{aligned}
$$

or in vector form:

$$
\begin{aligned}
& \left\|\left[\begin{array}{l}
x_{\mathrm{r}}(n, m) \\
x_{\mathrm{g}}(n, m) \\
x_{\mathrm{b}}(n, m)
\end{array}\right]-\left[\begin{array}{c}
189.61 \\
114.6 \\
1.4
\end{array}\right]\right\|^{2} \leqslant\left\|\left[\begin{array}{l}
x_{\mathrm{r}}(n, m) \\
x_{\mathrm{g}}(n, m) \\
x_{\mathrm{b}}(n, m)
\end{array}\right]-\left[\begin{array}{c}
198.43 \\
159.53 \\
65.12
\end{array}\right]\right\|^{2} \\
& \left\|\left[\begin{array}{l}
x_{\mathrm{r}}(n, m) \\
x_{\mathrm{g}}(n, m) \\
x_{\mathrm{b}}(n, m)
\end{array}\right]-\left[\begin{array}{c}
189.61 \\
114.6 \\
1.4
\end{array}\right]\right\|^{2} \leqslant\left\|\left[\begin{array}{l}
x_{\mathrm{r}}(n, m) \\
x_{\mathrm{g}}(n, m) \\
x_{\mathrm{b}}(n, m)
\end{array}\right]-\left[\begin{array}{c}
103.56 \\
41.75 \\
26.18
\end{array}\right]\right\|^{2}
\end{aligned}
$$

Members of Set 1 and Set 3 are defined in a similar manner. 
Table 1

Cropped window pixel values of Regions 1, 2 and 3 shown in Fig. 2a

\begin{tabular}{|c|c|c|c|c|c|c|c|c|c|c|c|c|c|c|c|c|c|c|c|c|c|c|c|c|c|c|c|c|c|}
\hline \multicolumn{10}{|c|}{ Red component } & \multicolumn{10}{|c|}{ Green component } & \multicolumn{10}{|c|}{ Blue component } \\
\hline \multicolumn{30}{|c|}{ Region 1} \\
\hline 198 & 201 & 202 & 201 & 201 & 201 & 199 & 199 & 198 & 198 & 156 & 157 & 162 & 159 & 160 & 160 & 157 & 156 & 155 & 154 & 24 & 38 & 49 & 60 & 65 & 65 & 65 & 65 & 69 & 10 \\
\hline 197 & 198 & 202 & 202 & 202 & 201 & 199 & 199 & 198 & 198 & 159 & 159 & 161 & 161 & 160 & 160 & 157 & 157 & 155 & 155 & 26 & 46 & 58 & 64 & 72 & 72 & 72 & 71 & 71 & 71 \\
\hline 199 & 199 & 202 & 202 & 204 & 201 & 199 & 199 & 199 & 199 & 159 & 159 & 163 & 165 & 165 & 160 & 160 & 159 & 157 & 157 & 39 & 51 & 61 & 68 & 72 & 72 & 74 & 74 & 76 & 77 \\
\hline 200 & 199 & 199 & 201 & 203 & 201 & 199 & 199 & 198 & 200 & 157 & 157 & 160 & 165 & 165 & 165 & 162 & 159 & 159 & 159 & 39 & 46 & 60 & 66 & 68 & 71 & 71 & 74 & 78 & 78 \\
\hline 200 & 200 & 198 & 197 & 198 & 199 & 199 & 201 & 199 & 198 & 158 & 158 & 159 & 160 & 162 & 164 & 164 & 164 & 162 & 162 & 43 & 54 & 56 & 60 & 64 & 68 & 71 & 75 & 78 & 78 \\
\hline 200 & 200 & 195 & 195 & 195 & 196 & 199 & 197 & 197 & 198 & 157 & 157 & 157 & 157 & 160 & 160 & 164 & 162 & 161 & 161 & 45 & 54 & 57 & 59 & 60 & 64 & 71 & 73 & 76 & 78 \\
\hline 201 & 200 & 195 & 194 & 190 & 189 & 195 & 195 & 196 & 196 & 159 & 159 & 157 & 156 & 152 & 153 & 159 & 159 & 161 & 161 & 57 & 59 & 59 & 59 & 59 & 63 & 71 & 73 & 76 & 79 \\
\hline 204 & 202 & 198 & 192 & 189 & 189 & 193 & 195 & 196 & 196 & 164 & 163 & 157 & 154 & 152 & 153 & 157 & 159 & 159 & 159 & 64 & 66 & 62 & 61 & 59 & 62 & 69 & 75 & 79 & 79 \\
\hline 207 & 205 & 200 & 193 & 192 & 192 & 198 & 198 & 197 & 195 & 165 & 164 & 160 & 156 & 154 & 154 & 161 & 161 & 160 & 157 & 67 & 67 & 66 & 63 & 61 & 61 & 67 & 76 & 79 & 79 \\
\hline 206 & 205 & 203 & 198 & 193 & 196 & 202 & 203 & 203 & 195 & 167 & 165 & 163 & 157 & 156 & 161 & 165 & 168 & 168 & 156 & 71 & 72 & 63 & 61 & 61 & 66 & 69 & 79 & 79 & 79 \\
\hline \multicolumn{30}{|c|}{ Region 2} \\
\hline 189 & 188 & 188 & 189 & 189 & 192 & 198 & 199 & 203 & 199 & 114 & 112 & 111 & 112 & 112 & 1113 & 116 & 117 & 116 & 115 & 3 & 3 & 3 & 3 & 3 & 1 & 4 & 9 & 10 & 3 \\
\hline 189 & 188 & 188 & 188 & 189 & 191 & 192 & 197 & 199 & 197 & 114 & 114 & 114 & 115 & 115 & 115 & 115 & 115 & 115 & 115 & 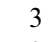 & 3 & 3 & 3 & 1 & 0 & 0 & 1 & 3 & 3 \\
\hline 189 & 188 & 188 & 188 & 188 & 190 & 190 & 193 & 197 & 196 & 117 & 114 & 114 & 115 & 116 & 115 & 115 & 113 & 115 & 115 & 3 & 1 & 1 & 1 & 1 & 0 & 0 & 0 & 3 & 4 \\
\hline 189 & 187 & 187 & 188 & 188 & 188 & 188 & 188 & 190 & 194 & 117 & 116 & 116 & 117 & 117 & 115 & 114 & 112 & 112 & 116 & 1 & 0 & 1 & 1 & 1 & 0 & 0 & 0 & 0 & 4 \\
\hline 188 & 187 & 187 & 187 & 188 & 188 & 187 & 188 & 188 & 189 & 117 & 117 & 117 & 118 & 116 & 115 & 112 & 112 & 112 & 114 & 0 & 0 & 0 & 1 & 1 & 0 & 0 & 0 & 0 & 1 \\
\hline 190 & 187 & 188 & 188 & 188 & 187 & 187 & 187 & 187 & 188 & 120 & 118 & 119 & 118 & 117 & 114 & 111 & 111 & 111 & 113 & ) & 0 & 0 & 2 & 2 & 0 & 0 & 0 & 0 & 0 \\
\hline 190 & 189 & 188 & 187 & 188 & 187 & 186 & 186 & 187 & 189 & 120 & 118 & 118 & 118 & 11 & 113 & 110 & 110 & 111 & 114 & ) & 0 & 0 & 0 & 3 & 1 & 1 & 1 & 1 & 1 \\
\hline 191 & 189 & 188 & 187 & 187 & 187 & 187 & 187 & 18 & 190 & 118 & 116 & 116 & 114 & 1 & 1 & 111 & 111 & 113 & 1 & 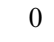 & 0 & 0 & 0 & 4 & 1 & 1 & 1 & 1 & 3 \\
\hline 191 & 189 & 188 & 188 & 188 & 188 & 188 & 189 & 190 & 191 & 11 & 116 & 114 & 113 & 1 & 1 & 112 & 113 & 114 & 116 & ) & 0 & 0 & 0 & 3 & 4 & 3 & 1 & 1 & 3 \\
\hline 195 & 193 & 189 & 189 & 189 & 190 & 193 & 192 & 191 & 191 & 115 & 115 & 114 & 114 & 113 & 113 & 116 & 117 & 116 & 117 & 1 & 0 & 0 & 2 & 3 & 3 & 3 & 1 & 0 & 0 \\
\hline \multicolumn{30}{|c|}{ Region 3} \\
\hline 125 & 125 & 112 & 97 & 97 & 102 & 106 & 106 & 106 & 107 & 5 & 5 & 48 & 48 & 4 & 5 & 44 & 4 & 44 & 50 & 29 & 29 & 29 & 29 & 31 & 27 & 22 & 22 & 23 & 30 \\
\hline 129 & 129 & 112 & 102 & 97 & 97 & 105 & 108 & 114 & 114 & 7 & 78 & 63 & 55 & 43 & 43 & 43 & 47 & 50 & 52 & 59 & 59 & 69 & 58 & 43 & 31 & 27 & 27 & 26 & 25 \\
\hline 141 & 153 & 153 & 105 & 90 & 90 & 92 & 114 & 115 & 115 & 7 & 109 & 109 & 55 & 33 & 31 & 31 & 47 & 54 & 55 & 62 & 89 & 89 & 63 & 46 & 27 & 27 & 28 & 28 & 26 \\
\hline 113 & 118 & 100 & 90 & 85 & 92 & 102 & 115 & 115 & 11 & 6 & 7 & 39 & 33 & 32 & 32 & 37 & 52 & 55 & 59 & 17 & 43 & 43 & 47 & 43 & 22 & 22 & 22 & 24 & 25 \\
\hline 97 & 100 & 96 & 9 & 90 & 102 & 117 & 115 & 117 & 11 & 38 & 35 & 38 & 32 & 32 & 37 & 52 & 52 & 56 & 62 & 10 & 18 & 23 & 44 & 47 & 47 & 24 & 22 & 24 & 25 \\
\hline 96 & 96 & 9 & 9 & 92 & 102 & 113 & 115 & 117 & 11 & 3 & 3 & 38 & 34 & 34 & 37 & 44 & 52 & 60 & 75 & 8 & 18 & 22 & 23 & 22 & 19 & 24 & 24 & 30 & 30 \\
\hline 96 & 97 & 96 & 90 & 90 & 90 & 96 & 9 & 118 & 132 & 35 & 42 & 38 & 29 & 16 & 18 & 20 & 35 & 62 & 81 & 12 & 18 & 20 & 12 & 12 & 7 & 13 & 20 & 31 & 55 \\
\hline 97 & 101 & 94 & 90 & 90 & 92 & 96 & 96 & 97 & 132 & 42 & 43 & 34 & 18 & 15 & 16 & 20 & 26 & 35 & 75 & 12 & 20 & 20 & 7 & 2 & 7 & 12 & 12 & 20 & 55 \\
\hline 102 & 104 & 94 & 90 & 92 & 95 & 96 & 96 & 92 & 97 & 43 & 43 & 30 & 17 & 16 & 18 & 20 & 25 & 26 & 31 & 20 & 28 & 18 & 7 & 5 & 7 & 7 & 12 & 12 & 15 \\
\hline 105 & 105 & 94 & 90 & 90 & 95 & 92 & 87 & 79 & 92 & 43 & 43 & 30 & 17 & 13 & 17 & 17 & 19 & 9 & 9 & 32 & 32 & 32 & 7 & 4 & 7 & 4 & 7 & 4 & 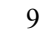 \\
\hline
\end{tabular}


The potato chip image was processed, pixel by pixel, to determine the entries of Sets 1, 2 and 3. This process is also called vector quantization (Cetin \& Weerackody, 1988; Rabiner \& Juang, 1993) and the corresponding MATLAB code is given in Appendix A. The segmented image is shown in Fig. 2b. In this image, Set 2 pixels occupy 55\% of the area of the entire image. The normalized area of Set 2 (NA2) was defined as the number of pixels in Set 2 divided by the number of pixels in the entire potato chip image. It was experimentally observed that there was a strong correlation between NA2 values and the measured acrylamide levels. As shown in Fig. 3, both the normalized area of Set 2 and the normalized acrylamide concentration of potato chips follow the same pattern during the frying process.

Image segmentation method, mentioned above, is semiautomatic and depends on the selection of representative RGB means of three regions before the segmentation process starts. Automatic segmentation can also be carried out using a public domain mean shift-based segmentation tool (Comaniciu \& Meer, 2002). The mean shift segmentation algorithm was initialized with the following parameters: Spatial bandwidth, 10 pixels and Colour bandwidth, 15 pixels. Using the mean shift segmentation algorithm, similar results were obtained, as shown in Fig. 2c. In this image, Set 2 pixels occupy $59 \%$ of the area of the entire image. Segmentation results of automatic or semi-automatic methods were close to each other.

In order to predict the acrylamide concentration of a given potato chip sample, a calibration curve of the NA2 parameter versus the measured acrylamide concentration was plotted, using the kinetic data obtained for potato chips prepared by frying at $170{ }^{\circ} \mathrm{C}$. As can be seen in this plot, shown in Fig. 4, there is a clear linear correlation between the acrylamide levels and the NA2 parameter.

\subsection{Test of the applicability of computer vision-based analysis approach}

The applicability of this approach for the prediction of acrylamide level in fried potatoes was verified by analyzing a number of commercial and home-made fried potato sam-

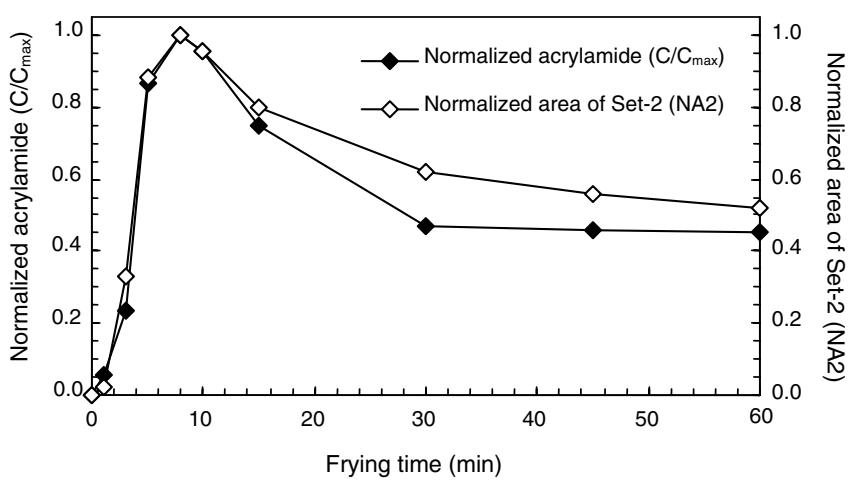

Fig. 3. Change of normalized acrylamide and NA2 values in potato chips during frying.

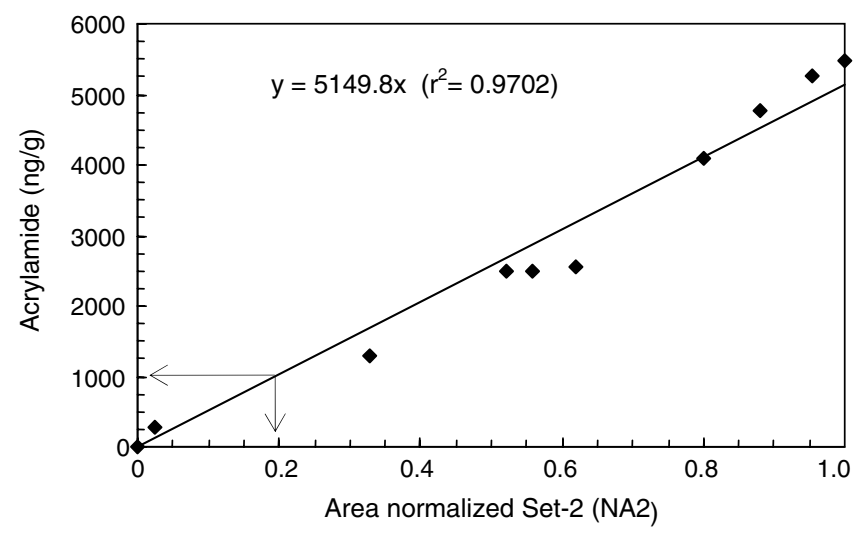

Fig. 4. Correlation between the NA2 parameter and the acrylamide level in fried potato chips.

ples. Measured and predicted acrylamide concentrations of commercial potato chips $(n=3)$ and home-made french fries $(n=10)$ are given in Table 2 . The linear regression equation $(y=5149.8 x)$ was used to calculate the acrylamide concentration of a potato sample from the NA2 parameter determined by computer vision-based image analysis. Measured and predicted values of acrylamide concentrations compare well with each other. Mean difference between the measured and the predicted acrylamide concentrations were $+4 \pm 14 \%$ and $-14 \pm 24 \%$ for commercial potato chips and home-made french fries, respectively. In both potato chips and french fries, only a randomly selected single face of a given sample was used to estimate the NA2 parameter, and thus to estimate acrylamide concentration. Although it is more reliable to determine a volumetric parameter for the estimation of acrylamide level, this is not possible from two dimensional digital images. It would be more accurate to analyze every face of a given french fry but in a computerized machine vision classification system; it is also not practical to image six faces of a fried potato.

Table 2

Measured and predicted acrylamide concentrations of commercial potato chips and home-made french fries

\begin{tabular}{lcccl}
\hline & NA2 & \multicolumn{2}{c}{ Acrylamide $(\mathrm{ng} / \mathrm{g})$} & Difference $^{\mathrm{a}}(\%)$ \\
\cline { 2 - 4 } & & Predicted & Measured & \\
\hline Potato chips & 0.15 & 782 & 897 & -13 \\
& 0.37 & 1883 & 1675 & +12 \\
French fries & 0.55 & 2834 & 2543 & +11 \\
& 0.57 & 2922 & 4849 & -40 \\
& 0.28 & 1464 & 2445 & -40 \\
& 0.66 & 3409 & 6151 & -45 \\
& 0.24 & 1218 & 2063 & -41 \\
& 0.49 & 2515 & 3636 & -31 \\
& 0.18 & 931 & 684 & +36 \\
& 0.05 & 245 & 332 & -26 \\
& 0.21 & 1061 & 1392 & -24 \\
& 0.04 & 183 & 200 & -8 \\
& 0.18 & 941 & 1053 & -11 \\
\hline
\end{tabular}

${ }^{\mathrm{a}}$ Difference $=\frac{\text { Predicted }- \text { Measuured }}{\text { Measured }} \times 100$. 


\section{Conclusion}

The computer vision-based image analysis seems to be a promising approach for the prediction of acrylamide level in fried potatoes. Since higher NA2 values indicate higher acrylamide levels, fried potatoes, exceeding a predefined critical value of NA2, may be simply sorted out in a processing line based on this principle. In such systems, cameras can be installed in the packaging lines and fried potato images can be analyzed in real time and those products with high NA2 values can be removed. For example, if a provisional maximum permitted concentration of acrylamide in the finished product is established as $1000 \mathrm{ng} / \mathrm{g}$, the fried potatoes exceeding a NA2 value of 0.2 would be removed by the machine vision system.

It should be noted that the calibration would need to be developed using more replicates over a wider range of concentrations, potato cultivars and frying conditions to improve accuracy of the approach. The purpose of this report was to demonstrate the feasibility of the method, not to implement it.

\section{Acknowledgements}

We thank the Turkish Academy of Sciences (GEBIP Study Grant) for financial support, TUBITAK Ankara Test and Analysis Laboratory (ATAL) for LC-MS analyses and Waters Corporation and Agilent Technologies for supplying some consumables.

\section{Appendix A}

Function VectorQuantize accepts two inputs: im_seq (image to be segmented) and $u$ (reference means) Representative cluster centers for Region 1, Region 2 and Region 3 are held in vector $u$ together with an additional cluster center for background. This extra fourthvalue is to separate the background from potato image. Segmentation is carried on the image held in im seq by using a nearest mean classifier. AN2 value is printed to the command window at the program termination. Segmented image can be displayed using the following code.

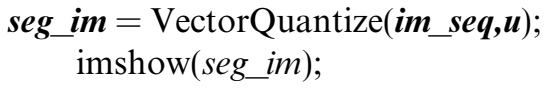

$\%$ File VectorQuantize.m

function $\left[\operatorname{seg} \_\right.$im $]=$VectorQuantize $($im_seg,, $\mathrm{u})$;

$[\mathrm{r}$ c h $]=$ size $\left(\mathrm{im} \_\right.$seg $)$;

$\%$ median filter the segmentation image with a $[3 \times 3]$ window to remove tiny oil sparks

filt $\_$im $=\operatorname{cat}\left(3\right.$, medfilt $2\left(\mathrm{im} \_\operatorname{seg}(:,:, 1)\right.$, 'symmetric'),medfilt2(im_seg(:,:, 2), . . 'symmetric'),medfilt2(im_seg(:,:,3), 'symmetric'));

$\%$ reduce from 3 dimensions to 2 dimensions for easy handling of data im = reshape(filt_im, r $\left.{ }^{*} \mathrm{c}, \mathrm{h}\right)$ ';
$\%$ compute the distance from cluster centers for all pixels

for $\mathrm{i}=1: 4$

$\operatorname{dist}(\mathrm{i},:)=\operatorname{sum}\left(\left(\operatorname{im}-\operatorname{repmat}\left(\mathrm{u}(:, \mathrm{i}),\left[1 \mathrm{r}^{*} \mathrm{c}\right]\right)\right) \div 2\right)$;

end

$\%$ find and store the location of minimum distance cluster for each pixel

$[\mathrm{y}$ loc $]=\min (\operatorname{dist}) ;$ vseg im $=\operatorname{zeros}\left(\mathrm{r}^{*} \mathrm{c}, \mathrm{h}\right)$;

$\%$ change pixels values with their representative cluster means for displaying purposes

for $\mathrm{i}=1: 4$

$$
\begin{aligned}
& \text { pos }=\text { find }(\text { loc }==\mathrm{i}) \\
& \operatorname{seg} \operatorname{im}(\operatorname{pos},:)=\operatorname{repmat}\left(\mathrm{u}(:, \mathrm{i})^{\prime},[\text { length(pos) } 1]\right) ;
\end{aligned}
$$

end

$\%$ restore the image back to its original dimensions

seg im = reshape(seg im, [r c h]);

$\%$ median filter the segmented image with a [7×7] window to fuse tiny unconnected

$\%$ regions

seg im $=\operatorname{cat}(3$, medfilt $2(\operatorname{seg}$ im(:,:,1), [7 7], 'symmetric'), medfilt2(seg im(:,:,2), ․ [7 7], 'symmetric'), medfilt2(seg im(:,:,3),[7 7],'symmetric'));

$\%$ compute AN2 ratio from segmented image

ratio $=$ length $($ find $($ loc $==2)) /$ length $($ find $(\operatorname{loc} \sim=4)$ );

$\%$ display this ratio in command prompt

disp(ratio);

\section{References}

Amrein, T. M., Schönbächler, B., Escher, F., \& Amado, R. (2004). Acrylamide in gingerbread: critical factors for formation and possible ways for reduction. Journal of Agricultural and Food Chemistry, 52, 4282-4288.

Cetin, A. E., \& Weerackody, V. (1988). Design of vector quantizers using simulated annealing. IEEE Transactions on Circuits Systems, 35(12), 1550.

Comaniciu, D., \& Meer, P. (2002). Mean shift: a robust approach toward feature space analysis. IEEE Transactions on Pattern Analysis and Machine Intelligence, 24(5), 603-619.

Friedman, M. (2003). Chemistry, biochemistry, and safety of acrylamide: a review. Journal of Agricultural and Food Chemistry, 51, 4504-4526.

Gökmen, V., Şenyuva, H.Z., Acar, J., Sarığlu, K., 2005. Determination of acrylamide in potato chips and crisps by high-performance liquid chromatography. Journal of Chromatography $A$ (doi:10.1016/ j.chroma.2004.10.094).

Gonzales, R. C., \& Woods, R. E. (2002). Digital image processing. New Jersey: Prentice-Hall.

Márquez, G., \& Aňón, M. C. (1986). Influence of reducing sugars and amino acids in the color development of fried potatoes. Journal of Food Science, 51, 157-160.

Martins, S. I. F. S., \& van Boekel, M. A. J. S. (2003). Melanoidins extinction coefficient in the glucose/glycine Maillard reaction. Food Chemistry, 83(1), 135-142.

Mottram, D. S., Wedzicha, B. L., \& Dodson, A. T. (2002). Acrylamide is formed in the Maillard reaction. Nature, 419, 448-449.

Papadakis, S. E., Abdul-Malek, S., Kamdem, R. E., \& Yam, K. L. (2000). A versatile and inexpensive technique for measuring color of foods. Food Technology, 54(12), 48-51.

Pedrechi, F., Moyano, P., Kaack, K., \& Granby, K. (2005). Color changes and acrylamide formation in fried potato slices. Food Research International, 38, 1-9.

Rabiner, L., \& Juang, B. H. (1993). Fundamentals of speech recognition. New Jersey: Prentice Hall. 
Şenyuva, H. Z., \& Gökmen, V. (2005). Study of acrylamide in coffee using an improved liquid chromatography mass-spectrometry method: investigation of colour changes and acrylamide formation in coffee during roasting. Food Additives and Contaminants, 22(3), 214-220.

Stadler, R. H., Blank, I., Varga, N., Robert, F., Hau, J., Guy, P. A., Robert, M. C., \& Riediker, S. (2002). Acrylamide from Maillard reaction products. Nature, 419, 449-450.

Surdyk, N., Rosén, J., Andersson, R., \& Åman, P. (2004). Effects of asparagine, fructose, and baking conditions on acrylamide content in yeast-leavened wheat bread. Journal of Agricultural and Food Chemistry, 52, 2047-2051.

Taubert, D., Harlfinger, S., Henkes, L., Berkels, R., \& Schömig, E. (2004). Influence of processing parameters on acrylamide formation during frying of potatoes. Journal of Agricultural and Food Chemistry, 52, 2735-2739.

Yaylayan, V. A., Wnorowski, A., \& Locas, C. P. (2003). Why asparagine needs carbohydrates to generate acrylamide. Journal of Agricultural and Food Chemistry, 51, 1753-1757. 